\title{
Low-Titer Group O Whole-Blood Resuscitation in the Prehospital Setting in Israel: Review of the First 2.5 Years' Experience
}

\author{
Dan Levin $^{a}$ Maoz Zur ${ }^{b}$ Eilat Shinar ${ }^{c, d}$ Tzadok Moshe ${ }^{c, d}$ Avishai M. Tsur ${ }^{a, e}$ \\ Roy Nadler $^{\mathrm{a}, \mathrm{f}}$ Mark H. Yazer ${ }^{\mathrm{g}, \mathrm{h}}$ Danny Epstein ${ }^{\mathrm{a}, \mathrm{i}}$ Guy Avital ${ }^{\mathrm{a}, \mathrm{j}}$ Shaul Gelikas $^{\mathrm{a}}$ \\ Elon Glassberg ${ }^{a, k, l} \quad$ Avi Benov ${ }^{a, k}$ Jacob Chen ${ }^{a, m}$ \\ ${ }^{a}$ Trauma and Combat Medicine Branch, Israel Defense Forces Medical Corps, Tel Hashomer, Ramat Gan, Israel; \\ ${ }^{b}$ Military Medical Academy, Medical Corps, Israel Defense Forces, Shefayim, Israel; ' Magen David National Blood \\ Services, Ramat Gan, Israel; 'Faculty of Health Sciences, Ben Gurion University of the Negev, Be'er Scheva, Israel; \\ eDepartment of Medicine B, Sheba Medical Center, Tel Hashomer, affiliated with Sackler Faculty of Medicine, Tel

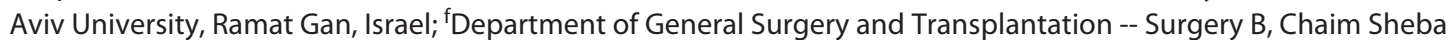 \\ Medical Center, Tel Hashomer, affiliated with the Sackler School of Medicine, Ramat Gan, Israel; 9Department of \\ Pathology, University of Pittsburgh, Pittsburgh, PA, USA; hDepartment of Pathology, Tel Aviv University, Tel Aviv, \\ Israel; 'Critical Care Division, Rambam Health Care Campus, Haifa, Israel; 'Division of Anesthesia, Intensive Care and \\ Pain Management, Tel Aviv Medical Center, Tel Aviv University, Tel Aviv, Israel; kThe Azrieli Faculty of Medicine, \\ Bar-llan University, Safed, Israel; 'The Uniformed Services, University of the Health Sciences, Bethesda, MD, USA; \\ mMeir Medical Center, affiliated with Sackler School of Medicine, Tel Aviv University, Kfar Saba, Israel
}

\section{Keywords \\ Whole blood · Low-titer O+ blood · Resuscitation · \\ Prehospital setting · Hemorrhagic shock}

\begin{abstract}
Introduction: The Israeli Defense Forces Medical Corps (IDF$\mathrm{MC}$ ) implemented the use of low-titer group $\mathrm{O}$ whole blood (LTOWB) as the first-choice resuscitation fluid in the IDF airborne Combat Search and Rescue Unit (IDF-CSAR) for aerial evacuation of both military and civilian casualties in June 2018 for injured patients with hemorrhagic shock and at least one of the following: systolic blood pressure $<90 \mathrm{~mm}$ $\mathrm{Hg}$, heart rate $>130$ beats/min, deterioration of consciousness without head injury or hemoglobin concentration $\leq 7 \mathrm{~g} /$ dL. Method: All casualties treated with LTOWB by IDF-CSAR providers from June 2018 to January 2021 were included. Demographic and prehospital treatment data were collected in order to check compliance and adherence to the IDFMC guidelines. This is a follow-up retrospective report. $R \boldsymbol{e}$ sults: Overall, 1,608 LTOWB units were supplied to the IDF-
\end{abstract}

\section{karger@karger.com} www.karger.com/tmh

Karger ${ }^{\prime \prime} \%$

BOPEN ACCESS
(C) 2021 The Author(s)

Published by S. Karger AG, Basel

This is an Open Access article licensed under the Creative Common Attribution-NonCommercial-4.0 International License (CC BY-NC) (http://www.karger.com/Services/OpenAccessLicense), applicable to the online version of the article only. Usage and distribution for commercial purposes requires written permission.
CSAR during the study period. Of these, 33 were transfused to 27 casualties; 17 (69\%) with blunt injury, 8 (29.6\%) with penetrating injuries, and $1(3.7 \%)$ with gastrointestinal bleeding without trauma. The leading cause of injury was motor vehicle accidents. A total of 23 casualties received 1 unit of LTOWB, 3 received 2 units and 1 patient received 4 units. Two casualties were children. The median heart rate was 120 beats/min, $8(29.6 \%)$ casualties had heart rates $>130$ beats/min. Median systolic blood pressure was $95 \mathrm{~mm} \mathrm{Hg}, 7$ (26\%) casualties had blood pressure $<90 \mathrm{~mm} \mathrm{Hg}$. The median Glasgow Coma Score was 14. No adverse reactions were documented following the administration of LTOWB. 77.8\% of patients received LTOWB in adherence to the guidelines. Conclusion: Appropriate administration of LTOWB has improved over time in IDF-CSAR. Using LTOWB is feasible and simpler than administering packed red blood cells and plasma concurrently. Further efforts are needed to introduce

D.L. and M.Z. contributed equally to the manuscript and are co-first authors. A.B. and J.C. contributed equally to the manuscript and are co-last authors. 
LTOWB in other prehospital and in-hospital scenarios, with an increase in the maximum antibody titer threshold, to meet the expected increase in demand.

(c) 2021 The Author(s).

Published by S. Karger AG, Basel

\section{Introduction}

Hemorrhage is the most common cause of preventable death in trauma and accounts for $25 \%$ of trauma deaths [1]. As soon as the bleeding is controlled, caregivers should try to restore circulating volume as quickly as possible. Early administration of blood components with varying ratios of packed red blood cells (PRBC) to plas$\mathrm{ma} /$ platelets showed better outcomes compared with crystalloid administration [2-4]. All hospitals in Israel use blood components when transfusions are required. However, in the prehospital scenario, volume resuscitation with various blood components is still considered to be complicated, due to storage and regulatory limitations.

Information gathered in the past 2 decades indicates that crystalloid-based resuscitation may be harmful to casualties [5-8]. Other studies showed that administration of PRBC or plasma in the prehospital environment improves outcomes [9-11]. Moreover, casualties who were infused with PRBC and plasma had higher survival rates, as compared to those infused with only 1 component, and especially compared to those casualties who received saline alone $[12,13]$.

The Israel Defense Forces Airborne Combat Search and Rescue Unit (IDF-CSAR) is the only entity in Israel with the ability to provide blood components to casualties in the prehospital scenario [14]. In 2013, the Israel Defense Forces Medical Corps (IDF-MC) adopted freezedried plasma as the primary volume resuscitation fluid for trauma casualties in field units [15-17]. While evidence shows that the use of PRBC and plasma is beneficial, the logistics in terms of the number of bags, storage conditions, and others are cumbersome and limiting in the field. Since June 2018, low-titer group O whole blood (LTOWB) has been the fluid of choice for resuscitating hemorrhagic casualties in the IDF-CSAR [18]. While reports that demonstrate the safety and efficacy of prehospital plasma transfusion are accumulating [13, 19-21], few data are available regarding using LTOWB for prehospital resuscitation of trauma casualties with massive hemorrhage.

In this study, we reviewed the first 2.5 of LTOWB implementation and treatment in the IDF-MC and estimated the IDF-CSAR's adherence to written protocols for LTOWB administration and its safety when transfused to massively bleeding casualties.

\section{Methods}

\section{Study Design}

The IDF-MC ethical review board approved this registrybased, retrospective study (No. 2014-1948). The manuscript was written and edited according to the STROBE guidelines [22]. This is a retrospective study that reports on our extended experience of transfusing LTOWB in the prehospital setting [18].

\section{Study Population}

We extracted demographic and clinical data on all casualties who received at least 1 unit of LTOWB, both military and nonmilitary, from IDF trauma registry (IDF-TR) from June 2018 to January 2021.

\section{The Israel Defense Forces Trauma Registry}

The IDF-TR is a registry established in 1997 to collect prehospital data of injured casualties (military, civilian, or foreign) to whom medical teams from the IDF-MC provided care [23]. During the treatment, the teams fill out a casualty card. Within $24 \mathrm{~h}$ of completing treatment, the data on these cards are entered into a digital, web-based trauma registry by dedicated staff of the Trauma and Combat Medicine Branch in the IDF Surgeon General's Headquarters. This was the primary registry analyzed in this study.

\section{Variables}

Demographics, point of injury, and prehospital data extracted from the IDF-TR included date of injury, whether the incident was combat related, casualty's age and sex, urgency level, mechanisms of injury, injured body regions, vital signs and life-saving interventions. Data regarding adverse transfusion-related complications were also extracted. The mechanism of injury was categorized as penetrating, blunt, or other. Life-saving interventions included tourniquet application, hemostatic packing, endotracheal intubation, cricothyroidotomy and administration of crystalloids, freezedried plasma, PRBC, tranexamic acid, or anesthesia.

\section{LTOWB Preparation, Transportation, and Storage}

The LTOWB used in the Israeli whole-blood program is type $\mathrm{O}$ Rh positive collected from volunteer blood donors by the $\mathrm{Na}$ tional Magen David Adom Blood Services (MDABS).

A new protocol for titering anti-A and anti-B in group O-positive male donors was created, using automated blood group typing equipment (PK7300, Beckman-Coulter, USA), for the purpose of identifying and building a database of suitable LTOWB donors. When the program began, the critical titer of anti- $\mathrm{A}$ and $-\mathrm{B}$ for the LTOWB units was chosen as the equivalent of $<50$, as determined by a manual saline tube method with 5 -min room temperature incubation, 30-s centrifugation, and manually inspected for agglutination. This is the laboratory's reference method.

A plasma dilution of $1: 18$ on the $P K 7300$, incubated at $25^{\circ} \mathrm{C}$ for $1 \mathrm{~h}$ with in-house produced reagent $\mathrm{A} 1 \mathrm{~B}$ red blood cells, and also with a mix of commercially available A1 and B cells (Immucor, USA), was found to be the most comparable to the reference saline tube method described above. The A1B cells were used in addition to the commercially available reagent RBCs when performing the titer out of an abundance of caution.

Using these methods, about $15 \%$ of $500 \mathrm{O}$-positive samples diluted 1:18 and tested both on the PK7300 and the reference saline tube method at a 1:50 dilution did not demonstrate agglutination with the A1B RBC. These were therefore defined as LTOWB donors [24].

All whole-blood units in Israel are stored, immediately after collection, in butane-diol containing cooling trays (CompoCool I) or containers (CompoCool II, Fresnius, Germany), which lowers 
Table 1. Indications for administration of low-titer group O whole blood by IDF-MC guidelines (at least 1 criterion)

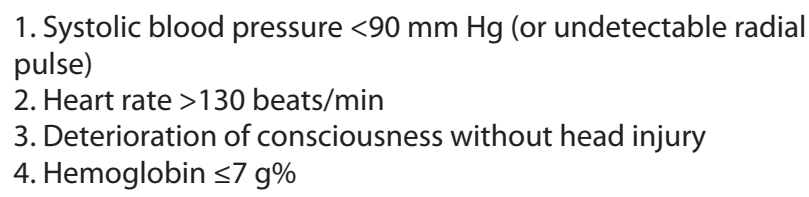

the blood units' temperature to $22 \pm 2^{\circ} \mathrm{C}$, thus allowing component processing within $22 \mathrm{~h}$ after collection [25]. Blood typing and antibody titration are performed after collection. Donations identified as low titer are tagged in the MDABS computer system and prevented from being separated into components, thus enabling the creation of the LTOWB inventory.

This conservative titer threshold $(<50)$ was initially selected to minimize the risk of hemolysis if administered to non-group $\mathrm{O}$ recipients. Moreover, as the AABB (formerly known as the American Association of Blood Banks) recommends, blood drawn only from male volunteers is used to lower the risk of transfusion-related acute lung injury. As the whole blood units are collected in CPDA-1 (Macopharma, France), the maximum shelf life of the LTOWB is 35 days, when stored between 2 and $8^{\circ} \mathrm{C}$. However, based on studies of platelet function in cold-stored whole blood, the maximum storage time of LTOWB, to be considered as a source of functional platelets, is limited to 21 days in the IDF [26].

In the IDF-CSAR unit the LTOWB units are kept in a refrigerator near the rescue equipment that is monitored with $\mathrm{Wi}$-fi based monitors and alarms. Before the IDF-CSAR helicopter team departs on a mission, an evaluation is made whether LTOWB is needed. Once decided that it might be needed, the LTOWB unit is carried in a PELICAN CREDO CUBE ${ }^{\mathrm{TM}}$ (Golden Hour ${ }^{\mathrm{TM}}$ ) that has been validated to maintain a temperature of $4^{\circ} \mathrm{C}$ for up to $48 \mathrm{~h}$.

\section{Whole-Blood Program in the IDF}

The whole-blood program was approved by the IDF's Surgeon General in May 2018. Since June 2018, when the Israeli wholeblood program started, each IDF-CSAR helicopter carries 2-3 units of LTOWB. When deployed, each IDF-CSAR helicopter team includes 2 providers trained in advanced life support, at least one of them is a flight surgeon, and several combat medics. During peacetime, the unit provides on-scene support for military medical providers, civilian emergency medical services (EMS), and interhospital patient transfers.

Indications for prehospital blood transfusion made according to the IDF-MC clinical practice guidelines regarding remote damage control resuscitation, which permit volume resuscitation, including blood, for casualties with at least 1 sign of profound hemorrhagic shock (Table 1). These guidelines have not changed since the LTOWB program started. We compared the casualties' variables to the clinical practice guidelines, in order to determine whether LTOWB was administered with adherence to guidelines.

\section{Results}

MDA Blood Services - Lessons Learned at the National Blood Center

From June 2018 to January 2021, MDABS screened 29,269 type O Rh-positive blood donors and found 3,171
Table 2. Demographics and injury characteristics of the LTOWB recipients in this study

\begin{tabular}{ll}
\hline Variable & Overall $(n=27)$ \\
\hline Age (median, IQR), years & $29.0(2,69)[6]$ \\
Children $(<8$ years old), $n(\%)$ & $2(7.4)$ \\
$\begin{array}{l}\text { Gender, } n(\%) \\
\text { Female }\end{array}$ & $5(18.5)$ \\
$\quad$ Male & $22(81.5)$ \\
Cause of injury, $n(\%)$ & $16(59.3)$ \\
$\quad$ Motor vehicle accident & $4(14.8)$ \\
Gunshot wound & $3(11.1)$ \\
Fall & $2(7.4)$ \\
$\quad$ Explosion & $1(3.7)$ \\
$\quad$ Helicopter crash & $1(3.7)$ \\
Gastrointestinal bleeding & $17(69)[1]$ \\
Mechanism of injury, $n(\%)$ & $8(29.6)$ \\
$\quad$ Blunt & $1(3.7)$ \\
Penetrating & \\
$\quad$ Other &
\end{tabular}

Missing data are reported in square brackets.

$(10.8 \%)$ donors with antibody titers $<50$. MDABS issued 3,040 LTOWB units for medical organization utilization. Of these, 1,771 (58.2\%) units were issued as LTOWB, 1,608 to the IDF-CSAR and 163 to Yoseftal Medical Center, a civilian hospital. The combined utilization rate of LTOWB by IDF-CSAR and Yoseftal Medical Center was $2.4 \%$, and 43 LTOWB units were transfused as such: 33 by IDF and 10 at the civilian hospital. The remaining 1,269 (41.8\%) LTOWB units, that were not issued, were manufactured by MDABS into PRBC units that were supplied to hospital blood banks nationwide.

\section{Disposition of Unused Units in the IDF-CSAR}

LTOWB units that are issued to the IDF-CSAR but are not used by day 21 are not returned to the blood center for the production of RBC units. These unused units are discarded. In total, as mentioned above, 1,608 LTOWB units have been issued to the IDF-CSAR and 33 have been transfused to patients by day 21 for a utilization rate of $2 \%$.

\section{Patient Demographics and Injury Characteristics}

During the study period, 27 casualties received 33 units of LTOWB. Most of the casualties were male ( $n=$ $22,81.5 \%)$. Two (7.8\%) children received LTOWB. The median age was 29 years (2-69), as shown in Table 2. Eight of these patients have been previously reported in a report of the Trauma and Combat Medicine Branch [18].

The injury mechanism was classified as blunt in 17 $(69 \%)$ casualties, penetrating in $8(29.6 \%)$ and other in 1 (3.4\%). Blunt injuries were mostly due to motor vehicle 
Table 3. Vital signs and life-saving interventions

\begin{tabular}{lll}
\hline Variable & Median (IQR) & Missing data \\
\hline Initial heart rate (range), beats/min & $120(38,180)$ & 1 \\
Initial heart rate $>130$ beats/min, $n$ (\%) & $8(29.6)$ & \\
Change in heart rate (range), beats/min & $4.5(-63,+59)$ & 3 \\
Initial systolic blood pressure (range), mm Hg & $95(54,130)$ & 6 \\
Initial systolic blood pressure $<90$ mm Hg, $n(\%)$ & $7(25.9)$ \\
Change in systolic blood pressure (range), mm Hg & $-11(-81,+73)$ & 12 \\
Initial GCS (range) & $14(3,15)$ \\
Change in GCS (range) & $-1(-12,+12)$ & 10 \\
Whole blood units administered, $n(\%)$ & \\
$<1$ & $11(40.7)$ \\
1 & $12(44.4)$ \\
2 & $3(11.1)$ \\
$>2$ & $1(3.8)$ \\
Pain control administered (morphine/ketamine), $n(\%)$ & $16(59.3)$ \\
Yes & $11(40.7)$ \\
No & $19(70.4)$ \\
Tranexamic acid administered, $n$ (\%) & $8(29.6)$ \\
Yes & $14(82.3)$ \\
No & $3(17.7)$ \\
Other fluid administered by IDF-CSAR (FDP/Hartman's), $n(\%)$ & \\
Yes & $5(18.5)$ \\
No & $1(3.7)$ \\
Airway management, $n$ (\%) & $4(14.8)$ \\
Positive pressure ventilation (w/mask) & $17(63)$ \\
Supraglottic device (LMA) & \\
Endotracheal tube & \\
No airway management & \\
\hline
\end{tabular}

GCS, Glasgow Coma Scale; FDP, freeze-dried plasma; IDF-CSAR, Israeli Defense Forces Airborne Combat Search and Rescue Unit; LMA, laryngeal mask airway. Change in heart rate/systolic blood pressure/GCS, the average change in the parameter from the first documented measurement to the last (around the hospital arrival). accidents or falls, and penetrating injuries were caused by gunshots or bomb explosions (Table 2).

The median initial heart rate was 120 beats/min (IQR $30-180)$. In 8 (29.6\%) casualties the initial heart rate was above 130 beats/min (Table 3 ). The initial median systolic blood pressure was $95 \mathrm{~mm} \mathrm{Hg}$ (IQR 54-130); it was below $90 \mathrm{~mm} \mathrm{Hg}$ in 7 (25.9\%) casualties. Data regarding the subsequent BP measurements were missing in 12 cases (44.4\%). The median initial GCS was 14 (IQR 3-15).

\section{Prehospital Life-Saving Interventions}

Table 3 summarizes the life-saving interventions utilized at the point of injury and during enroute care. Eleven (40.7\%) casualties, including 2 children, received fewer than 1 full unit of LTOWB during transportation to the hospital, $12(44.4 \%)$ casualties received 1 full unit, 3 (11.1\%) received 2 units. In 1 (3.4\%) case involving transfer from a rural hospital to a level I medical facility, the patient received 4 units. 19/27 (70.4\%) casualties received tranexamic acid. No adverse reactions were documented among LTOWB recipients, including clinical evidence for hemolytic transfusion reactions.

LTOWB Resuscitation in the Prehospital Setting

\section{Compliance to IDF-MC Clinical Practice Guidelines}

The IDF clinical practice guidelines state that resuscitation should only be administered in hemorrhagic shock, according to the indications described in Table 1. Comparing variables of each case to the clinical practice guidelines showed that IDF-CSAR's caregivers were in compliance in $21(77.8 \%)$ cases.

\section{Discussion}

LTOWB has been given by the IDF-CSAR unit since June 2018, as the resuscitation fluid of choice for hemorrhagic shock in the prehospital setting. By January 2021, 27 casualties had been transfused with LTOWB. Of these, $22.2 \%$ received treatment outside of the clinical practice guidelines, compared to our previous report, after the first year of LTOWB use, where $37.5 \%$ were inappropriately transfused [18]. Therefore, IDF-CSAR caregivers' adherence to the IDF-MC clinical practice guidelines has improved, most probably due to the efforts to ensure that LTOWB transfusion is implemented appropriately. How- 
ever, further education is required to improve and achieve full compliance.

The absence of hemolytic reactions is attributed to the very low titer threshold for anti-A and $-\mathrm{B}(<50)$. The small number of cases treated by the IDF-CSAR with LTOWB makes it impossible to make a clear conclusion regarding the safety of LTOWB administration. However, our results are in accord with other articles that demonstrate the safety of LTOWB $[27,28]$. Based on the accumulating data from the practices in other countries and our experience, MDABS is considering instituting a more permissive antibody titer threshold of $<100$, which will enable the blood center to more easily meet the increasing demand for LTOWB from both the pre- and in-hospital systems. This increased demand is largely based on a recent decision of the Israeli Trauma and Acute Care Surgery Council, that requires incorporating LTOWB in the massive hemorrhage protocols of small and remote hospitals, mostly for those who do not keep either pooled or single donor platelets in their inventories due to logistic and financial considerations. In addition, the use of LTOWB in emergency departments or trauma bays will be decided by trauma and transfusion medicine specialists in each hospital, and demand is expected to increase.

We documented 27 causalties who were treated with LTOWB during the observation period, which is a relatively small fraction of the total of 2,637 hemorrhagic shock patients treated in Israel between 2018 and 2020 . Most of these injured patients were treated by local paramedics who do not yet have access to LTOWB.

The main limitation of this study is the small number of LTOWB recipients. We intend to collect additional information regarding LTOWB administration in the prehospital setting and the outcomes of those casualties. Second, although IDF-CSAR is a military unit, most of the recent cases that have occurred in times of peace are civilian casualties. Yet, LTOWB should be applicable in military scenarios by IDF-CSAR in the same manner. However, since the major use of LTOWB has been mainly for civilian casualties, and since IDF-CSAR treats only a small fraction of the total civilian casualties, we assume that the utilization reported herein can inform and promote its use in civilian EMS that have not yet implemented an LTOWB program.

Another limitation of this work is that due to the nature of the database maintained by the IDF-CSAR, the registry includes only prehospital data, which explains why some patients appeared to receive only a partial unit of LTOWB. The transfusion of these units was completed once the patient arrived at the hospital. Furthermore, caregivers are given some leeway in deciding when to initiate a transfusion based on their clinical judgment, and this is not captured in the registry [14]. Therefore, we cannot explain all of the deviations from the LTOWB administration protocol.
One additional limitation to consider is the fact that the registry does not contain the recipient's blood group as this is determined once the patient arrives at the hospital. This is an important limitation in determining the serological safety of administering LTOWB to non-group O recipients; however, other reports have detailed its safety in adult and pediatric civilian patients [29-33], and no adverse events or clinical evidence of hemolysis were detected amongst the recipients in the current report.

Whole blood is widely considered as the optimal resuscitation fluid in hemorrhagic shock, with a substantial logistical advantage over separated PRBC and platelets in prehospital settings. Currently, whole blood provides the only feasible means of administering platelets in the prehospital setting. When compared to the common in-hospital practice of administration of blood components separately, LTOWB provides all blood components in a single bag, through a single intravenous line, thus shortening the time to transfusion of each individual component. Additionally, storage is simpler and transfusion itself requires fewer health care personnel, and balanced resuscitation can be administered quickly.

Since using LTOWB is safe and relatively simple, having it available in other prehospital platforms such as civilian ambulances should be considered. The Southwest Texas Regional Advisory Council's Regional Whole-Blood Program is particularly well described, where LTOWB units are placed in 30 end-point stations (helicopter EMS bases and ground EMS agencies) for 14 days, after which they are shipped to medical centers for another 3 weeks to reduce wastage (total of 35 days until the blood unit expires). After administration of 450 blood units, there were no prehospital complications documented, and the wastage (expiry) rate of blood units was 1-2\% [34, 35]. In Israel, we are limited in this context in that only physicians are authorized to approve blood component administration, whereas most caregivers in Israeli EMS systems are paramedics. Moreover, as stated, to preserve platelet function, our LTOWB units as such expire after 21 days.

Implementing LTOWB treatment as the resuscitation fluid of choice is still evolving in the IDF-CSAR. This reported a decrease in the number of cases where LTOWB was administered inappropriately without adherence to the IDF-MC guidelines compared to the first report of LTOWB use in the IDF-CSAR. Additional steps can be taken to improve the guidance and training of the teams. Furthermore, IDF-CSAR's early experience with LTOWB is promising, and expansion of the LTOWB program to other prehospital medical teams should be considered. We hope that, as has been the case of advanced tourniquets, freeze-dried plasma and other life-saving interventions, the experience learned by IDF-MC will lead to embrace LTOWB treatment by other civilian emergency care organizations. 


\section{Statement of Ethics}

The IDF-MC Institutional Review Board approved the study (No. 2014-1948) and waived the requirement for written informed consent.

\section{Conflict of Interest Statement}

D.L., M.Z., A.S., R.N., D.E., G.A., S.G., E.G., and A.B. are active military officers in the Israel Defense Force. This paper represents their research and reflects their personal insights that should not be attributed to the IDF-MC. For all authors, no conflicts of interest or funding were declared.

\section{Author Contributions}

Dan Levin: writing - original draft, investigation. Maoz Zur: writing - original draft, investigation. Eilat Shinar: review and editing. Tzadok Moshe: investigation. Avishai M. Tsur: review and editing. Roy Nadler: conceptualization, review and editing. Mark H. Yazer: review and editing. Danny Epstein: conceptualization, review and editing. Guy Avital: review and editing. Shaul Gelikas: review and editing. Elon Glassberg: review and editing. Avi Benov: review and editing. Jacob Chen: writing, conceptualization, review, and editing.

\section{Data Availability Statement}

Further data are available by request after approval granted by an IDF-MC committee.

\section{References}

1 Eastridge BJ, Mabry RL, Seguin P, Cantrell J, Tops T, Uribe P, et al. Death on the battlefield (2001-2011). J Trauma Acute Care Surg. 2012;73(6 Suppl 5): S431-7.

2 Holcomb JB, del Junco DJ, Fox EE, Wade CE, Cohen MJ, Schreiber MA, et al. The prospective, observational, multicenter, major trauma transfusion (PROMMTT) study. JAMA Surg. 2013;148:127-36.

3 Shaz BH, Dente CJ, Nicholas J, MacLeod JB, Young AN, Easley K, et al. Increased number of coagulation products in relationship to red blood cell products transfused improves mortality in trauma patients. Transfusion. 2010; 50:493-500.

4 Holcomb JB, Tilley BC, Baraniuk S, Fox EE, Wade CE, Podbielski JM, et al. Transfusion of plasma, platelets, and red blood cells in a 1:1: 1 vs a $1: 1: 2$ ratio and mortality in patients with severe trauma: the PROPPR randomized clinical trial. JAMA. 2015;313:471-82.

5 Lipsky AM, Ganor O, Abramovich A, Katzenell U, Glassberg E. Walking between the drops: Israeli defense forces' fluid resuscitation protocol. J Emerg Med. 2013;44:790-5.

6 Neal MD, Hoffman MK, Cuschieri J, Minei JP, Maier RV, Harbrecht BG, et al. Crystalloid to packed red blood cell transfusion ratio in the massively transfused patient: when a little goes a long way. J Trauma Acute Care Surg. 2012;72:892-8.

7 Driessen A, Fröhlich M, Schäfer N, Mutschler M, Defosse JM, Brockamp T, et al. Prehospital volume resuscitation - did evidence defeat the crystalloid dogma? An analysis of the Trauma Register DGU ${ }^{\circledast}$ 2002-2012. Scand J Trauma Resusc Emerg Med. 2016;24:42.

8 Bickell WH, Wall MJ, Pepe PE, Martin RR, Ginger VF, Allen MK, et al. Immediate versus delayed fluid resuscitation for hypotensive patients with penetrating torso injuries. $\mathrm{N}$ Engl J Med 1994;331:1105-9.

9 Shackelford SA, del Junco DJ, Powell-Dunford N, Mazuchowski EL, Howard JT, Kotwal RS, et al. Association of pre-hospital blood product transfusion during medical evacuation of combat casualties in Afghanistan with acute and 30day survival. JAMA. 2017;318:1581-91.
10 Griggs JE, Jeyanathan J, Joy M, Russell MQ, Durge N, Bootland D, et al. Mortality of civilian patients with suspected traumatic haemorrhage receiving pre-hospital transfusion of packed red blood cells compared to pre-hospital crystalloid. Scand J Trauma Resusc Emerg Med. 2018;26:100.

11 Barkana Y, Stein M, Maor R, Lynn M, Eldad A. Pre-hospital blood transfusion in prolonged evacuation. J Trauma. 1999;46:176-80.

12 Sperry JL, Guyette FX, Brown JB, Yazer MH, Triulzi DJ, Early-Young BJ, et al. Pre-hospital plasma during air medical transport in trauma patients at risk for hemorrhagic shock. N Engl J Med. 2018;379:315-26.

13 Guyette FX, Sperry JL, Peitzman AB, Billiar TR, Daley BJ, Miller RS, et al. Pre-hospital blood product and crystalloid resuscitation in the severely injured patient: a secondary analysis of the pre-hospital air medical plasma trial. Ann Surg. 2021;273(2):358-64.

14 Chen J, Benov A, Nadler R, Darlington DN, Cap AP, Lipsky AM, et al. Pre-hospital blood transfusion during aeromedical evacuation of trauma patients in Israel: the IDF CSAR experience. Mil Med. 2017;182(S1):47-52.

15 Glassberg E, Nadler R, Gendler S, Abramovich A, Spinella PC, Gerhardt RT, et al. Freezedried plasma at the point of injury: from concept to doctrine. Shock. 2013;40:444-50.

16 Shlaifer A, Siman-Tov M, Radomislensky I, Peleg K, Shina A, Baruch EN, et al. Pre-hospital administration of freeze-dried plasma, is it the solution for trauma casualties? J Trauma Acute Care Surg. 2017;83:675-82.

17 Glassberg E, Nadler R, Rasmussen TE, Abramovich A, Erlich T, Blackbourne LH, et al. Point-of-injury use of reconstituted freezedried plasma as a resuscitative fluid. J Trauma Acute Care Surg. 2013;75(2 Suppl 2):S111-4. 18 Nadler R, Tzur AM, Yazer MH, Shinar E, Moshe T, Benov A, et al. Early experience with transfusing low titer group $\mathrm{O}$ whole blood in the pre-hospital setting in Israel. Transfusion. 2020;60;S10-S16.
19 Shackelford SA, Del Junco DJ, Powell-Dunford N, Mazuchowski EL, Howard JT, Kotwal RS, et al. Association of prehospital blood product transfusion during medical evacuation of combat casualties in Afghanistan with acute and 30-day survival. JAMA. 2017; 318(16):1581-91.

20 Sperry JL, Guyette FX, Brown JB, Yazer MH, Triulzi DJ, Early-Young BJ, et al. Prehospital plasma during air medical transport in trauma patients at risk for hemorrhagic shock. $\mathrm{N}$ Engl J Med. 2018;379(4):315-26.

21 Rehn M, Weaver A, Brohi K, et al. Effect of prehospital red blood cell transfusion on mortality and time of death in civilian trauma patients. Shock. 2019;51(3):284-8

22 Vandenbroucke JP, von Elm E, Altman DG, Gøtzsche PC, Mulrow CD, Pocock SJ, et al. Strengthening the Reporting of Observational Studies in Epidemiology (STROBE): explanation and elaboration. PLoS Med. 2007 Oct; 4(10):e297.

23 Tsur AM, Nadler R, Lipsky AM, Levi D, Bader $\mathrm{T}, \mathrm{Benov} \mathrm{A}$, et al. The Israel Defense Forces Trauma Registry: 22 years of point-of-injury data. J Trauma Acute Care Surg. 2020 Aug; 89(2S Suppl 2):S32-S38.

24 Moshe T, Muncher L, Rushkin G, GoldmanLevi R, Yazer M, Yahalom V, et al. Determination of anti A and anti B titers in group $\mathrm{O}$ whole blood donors using an automated instrument, Transfusion. 2018 Sept;58 Suppl 2: S53A.

25 Shinar E, Etlin S, Frenkel O, Yahalom V. The implementation of rapid cooling and overnight hold of whole blood at ambient temperature before processing into components in Israel. Transfusion. 2011 Jan;51 Suppl 1:58S64S.

26 Pidcoke HF, McFaul SJ, Ramasubramanian AK, Parida BK, Mora AG, Fedyk CG, et al. Primary hemostatic capacity of whole blood: a comprehensive analysis of pathogen reduction and refrigeration effects over time. Transfusion. 2013 Jan;53(01):137s-149s. 
27 Malkin M, Nevo A, Brundage SI, Schreiber M. Effectiveness and safety of whole blood compared to balanced blood components in resuscitation of hemorrhaging trauma patients - a systematic review. Injury. 2021 Feb;52(2) 182-8.

28 Dishong D, Cap AC, Holcomb J, Triulzi DJ, Yazer $\mathrm{MH}$. The rebirth of the cool: a narrative review of the clinical outcomes of cold stored low titer group $\mathrm{O}$ whole blood recipients compared to conventional component recipients in trauma. Hematology.

29 Morgan K, Yazer MH, Triulzi, DJ, Strotmeyer S, Gaines B, Leeper C. Safety profile of low titer group $\mathrm{O}$ whole blood in pediatric patients with massive hemorrhage. Transfusion. 2021;61:S8-S14.
30 Harrold I, Seheult JS, Alarcon A, Sperry J, Triulzi DJ, Yazer MH. Hemolytic markers following the transfusion of uncrossmatched, cold-stored, low-titer, group $\mathrm{O}+$ whole blood in civilian trauma patients. Transfusion. 2020; 60 Suppl 3:S24-S30.

31 Seheult JN, Bahr M, Anto V, Alarcon LH, Corcos A, Sperry JL, et al. Safety profile of uncrossmatched, low-titer, group $\mathrm{O}+$ whole blood in civilian trauma patients. Transfusion. 2018;58:2280-8.

32 Seheult JN, Triulzi DJ, Alarcon LH, Sperry JL, Murdock A, Yazer MH. Measurement of hemolysis markers following transfusion of uncrossmatched, low-titer, group $\mathrm{O}+$ whole blood in civilian trauma patients: initial experience at a level 1 trauma center. Transfusion Med. 2017; 27:30-5
33 Yazer MH, Jackson B, Sperry J, Alarcon L, Triulzi DJ, Murdock A . Initial safety and feasibility of cold stored uncrossmatched whole blood transfusion in civilian trauma patients. J Trauma Acute Care Surg. 2016 Jul;81:21-6.

34 Randall S, Tasia L, David W, Rena S, Eric E, Elizabeth W, et al. Operationalizing the deployment of low-titer O-positive whole blood within a regional trauma system. Military Med. 2021;186 Suppl 1:391.

35 Zhu CS, Pokorny DM, Eastridge BJ, Nicholson SE, Epley E, Forcum J, et al. Give the trauma patient what they bleed, when and where they need it: establishing a comprehensive regional system of resuscitation based on patient need utilizing cold-stored, low-titer $\mathrm{O}+$ whole blood. Transfusion. 2019;59;1429-38. 